\title{
Effect of Blended Montomollironite on Crystallization of Poly(vinylidene fluoride)
}

\author{
By Eiji YAMADA,${ }^{1}$ Akihiro NISHIOKA,${ }^{2}$ Hideshige SUZUKI, ${ }^{1}$ Go MURASAWA, ${ }^{1}$ \\ Ken MIYATA, ${ }^{1}$ Tomonori KODA, ${ }^{1, *}$ and Susumu IKEDA ${ }^{1}$
}

The effect of blend of nano-clay on Poly(vinylidene fluoride) (PVDF) crystallization was examined in PVDF/organo-clay (organically modified clay) nanocomposites and PVDF/natural-clay (non-modified clay) composites. These two types of composite materials were prepared by a melt compounding method using a twin-screw extruder. The composites were characterized by X-ray diffraction (XRD) and Differential Scanning Calorimeter (DSC) measurement. We found that the PVDF crystallizes as $\beta$-form crystal on clay surface of both composite systems. Results for films made by slow cooling from melt are one important evidence that crystallization mechanism of $\beta$-form PVDF in these nanocomposites is epitaxial growth on the surface of clay platelets. In addition, we predict PVDF crystallization behaviors which depend on degree of supercooling by comparing quenched and slowly cooled samples of the composite systems.

KEY WORDS: Poly(vinylidene fluoride) / Clay / Montmollironite / Nanocomposites / Nucleating Agent / Crystallization / Epitaxial Growth /

Poly(vinylidene fluoride) (PVDF) has three crystalline polymorphs, $\alpha-, \beta$-, and $\gamma$-form depending on crystallization conditions. $^{1-5}$ In these polymorphs, only the $\beta$-form which is composed of all-trans chains exhibits ferroelectricity. ${ }^{6-11}$ Electrical dipoles are generated between fluorine and hydrogen atoms in individual all-trans molecular chains. Their directions are parallel to the $\mathrm{b}$ axis (polar axis) in the $\beta$-form inducing bulk polarization. The $\beta$-form of pure PVDF film is typically prepared by uniaxially stretching the $\alpha$-form film. The $\alpha$-form film which is composed of trans-gauche-trans-gauche' (TGTG') chains is obtained by cooling from melt.

Recent development for processing of PVDF was denoted by Priya and Jog who first reported composite materials of PVDF and organo-clay (organically modified clay). ${ }^{12-14}$ They inferred from X-ray analysis for the composites that the crystal obtained without stretching was the $\beta$-form.

In previous paper, we reconfirmed this fact by using X-ray analysis and furthermore measured the electric displacement $D$ versus electric field $E(D-E)$ hysteresis loop. ${ }^{15}$ As a result, we found the ferroelectricity of non-stretched film of PVDF/clay nanocomposites with $2 \%$ of organo-clay weight fraction. The important point was that the film was non-stretched. Pure PVDF $\alpha$-from crystal is obtained by cooling from melt without any special treatment such as stretching and it does not show any ferroelectricity. In addition, we suggested that the crystallization mechanism of the PVDF $\beta$-form in PVDF/clay nanocomposites is an epitaxial growth on the surface of clay platelet induced by the interfacial charge-dipole interaction and lattice matching. ${ }^{15}$

In the present study, the effect of blending clay on PVDF crystallization was examined for PVDF/organo-clay nanocomposites and PVDF/natural-clay (non-modified clay) com- posites by using X-ray diffraction and differential scanning calorimetry (DSC). Crystal structure, melting and crystallization behaviors were examined.

\section{SAMPLE PREPARATION}

PVDF, product named KF polymer (grade: T\#850), was purchased from Kureha. The organo-clay used is dimethyl hydrogenated-tallow ammonium-modified montmorillonite (Cloisite 20A) which was prepared by ion-exchanging sodium montmorillonite with alkyl ammonium cations. The naturalclay used is non-modified montmorillonite (Cloisite $\mathrm{Na}^{+}$). These two clays were purchased from Southern Clay Products.

Blended samples of PVDF pellets and the organo-clay or natural-clay powder were prepared by melt compounding which was performed in a twin-screw extruder (KZW30TW Technovel, $\mathrm{L} / \mathrm{D}=45$ ). The screw speed was $500 \mathrm{rpm}$ and the compounding temperature was $200{ }^{\circ} \mathrm{C}$. The obtained pellets of the composites were dried for $24 \mathrm{~h}$ in a vacuum oven at $80^{\circ} \mathrm{C}$ to remove absorbed water. For the present work, fractions of organo-clay (Cloisite 20A) in PVDF matrix were 0.1, 0.5, 2, and $5 \%$ of total weight, and those of natural-clay (Cloisite $\mathrm{Na}^{+}$) were $2,5,7$, and $10 \%$.

Film fabrication in this study was by the compression of melted pellets in a hot press (Toyo Seiki Mini Test Press) at $220^{\circ} \mathrm{C}$ at pressure $15 \mathrm{MPa}$ for $5 \mathrm{~min}$. We prepared two types of films by changing cooling process. One is slowly cooling after the melt compression and the other is rapid quenching in ice water. The slowly cooled film was kept between the pressing plates after removal of pressure until the equipment went back to room temperature. We describe these processes as the slowly cooled and the quenched.

\footnotetext{
${ }^{1}$ Graduate School of Science and Engineering, Yamagata University, Yonezawa 992-8510, Japan

${ }^{2}$ Cooperative Research Center, Yamagata University, Yonezawa, Japan

*To whom correspondence should be addressed (Tel \& Fax: +81-238-26-3207, E-mail: koda @yz.yamagata-u.ac.jp).
} 
Non-stretched pure PVDF film was the quenched film made from pure PVDF pellets. Uniaxially stretched PVDF film was prepared by uniaxially stretching of the non-stretched pure PVDF film. The stretching ratio of the film was 5 times its original length. Thicknesses of the films for the present study ranged from 20 to $50 \mu \mathrm{m}$.

Present rule of naming samples is as follows. Non-stretched pure PVDF film is denoted by $\alpha$-form film. Uniaxially stretched pure PVDF film is denoted by uniaxially stretched $\beta$-form film. For composite films, sample notation $\mathrm{VDF} / \mathrm{XNz}$ is used, where VDF stands for PVDF. The $\mathrm{X}$ of $\mathrm{VDF} / \mathrm{XNz}$ is replaced for $\mathrm{O}$ when the sample contains organo-clay (Cloisite 20A). On the other hands, it is replaced for $\mathrm{N}$ when natural-clay (Cloisite $\mathrm{Na}^{+}$) is contained. The $\mathrm{N}$ of $\mathrm{VDF} / \mathrm{XNz}$ expresses fraction of clay in weight $\%$ and the $\mathrm{z}$ describes cooling scheme; the $\mathrm{z}$ is replaced by q for quenched samples, and by s for slowly cooled samples. For example, $\mathrm{VDF} / \mathrm{O} 2 \mathrm{q}$ is the quenched film of PVDF containing Cloisite 20A of weight fraction $2 \%$.

\section{CHARACTERIZATION}

X-Ray diffraction measurements were performed using an X-ray diffractometer (Rigaku Rint 2000) in a reflection mode with $\mathrm{Cu} \mathrm{K} \alpha$ radiation (wavelength $\lambda=1.54 \AA$ ) at a voltage of $40 \mathrm{kV}$ and a current of $30 \mathrm{~mA}$ at room temperature. The samples were scanned at a scanning speed of $4^{\circ} / \mathrm{min}$ under a diffraction angle $2 \theta$ ranging from 2.0 to $40.0^{\circ}$.

Thermal analysis was performed using the Differential Scanning Calorimeter (DSC) (Q-100, TA Instruments). The samples were scanned at a scanning speed of $1{ }^{\circ} \mathrm{C} / \mathrm{min}$ under temperature range from 10 to $220^{\circ} \mathrm{C}$.

We measured the 1st heating, the 1st cooling, and the 2nd heating. Samples for DSC measurement were obtained form quenched films. The 2 nd heating process was for understanding of slowly cooled samples. It is measured after the 1st cooling of the cooling rate $1^{\circ} \mathrm{C} / \mathrm{min}$.

\section{RESULTS}

\section{Pure PVDF and Slowly Cooled Composites}

Figure 1 shows the XRD profiles for PVDF $\alpha$-form and uniaxailly stretched $\beta$-form films. The reflection peaks of the PVDF $\alpha$-form at $2 \theta=17.9,18.4,20.0$, and $26.6^{\circ}$ respectively corresponds to the (100), (020), (110), and (021) planes. The reflection peak of PVDF $\beta$-form at $2 \theta=20.7^{\circ}$ corresponds to the (110) and (200) planes. Figure 2 shows DSC heating curve at heating rate of $1{ }^{\circ} \mathrm{C} / \mathrm{min}$. Melting temperatures of PVDF $\alpha$-form and uniaxially stretched $\beta$-form are indicated at 176.6 and $173.1{ }^{\circ} \mathrm{C}$, respectively.

Figures 3 and 4 show X-ray profiles and DSC heating curves for slowly cooled films of PVDF/organo-clay nanocomposites with $0.1,0.5,2$, and $5 \%$ of organo-clay weight fractions. In Figure 3 , we see peaks at $2 \theta=18.5^{\circ}$ and $20.1^{\circ}$. The peak at $2 \theta=18.5^{\circ}$ decreased with increasing clay content causing single peak profile similar to the $\beta$-form indicated by Figure 1 . We attribute the peak of $2 \theta=20.1^{\circ}$ to the PVDF $\beta$-form

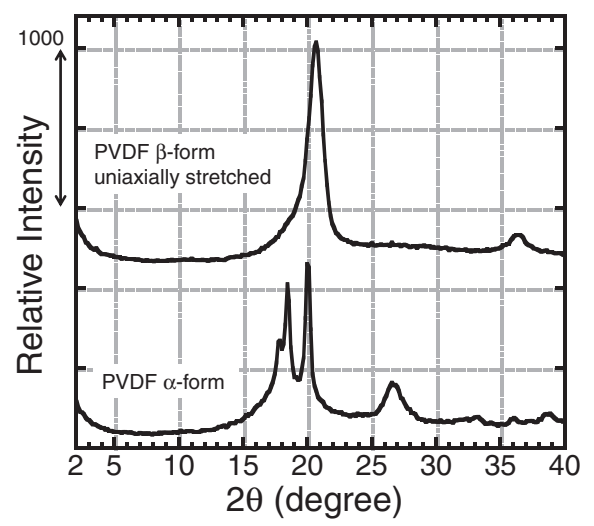

Figure 1. XRD profiles for pure PVDF uniaxially stretched $\beta$-form and $\alpha$-form.

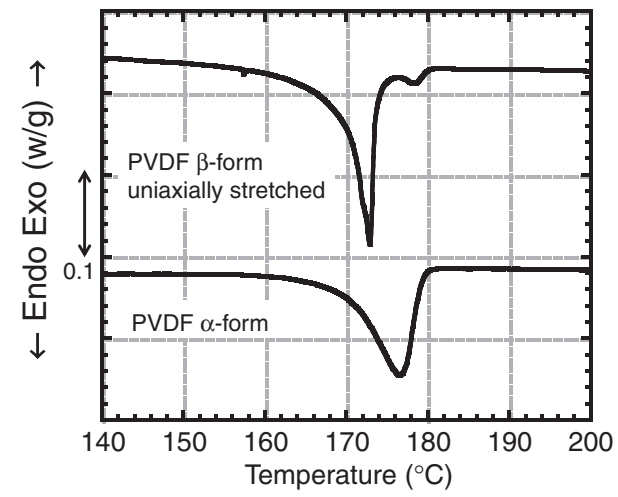

Figure 2. DSC heating curves at rate $1^{\circ} \mathrm{C} / \mathrm{min}$ for pure PVDF uniaxially stretched $\beta$-form and $\alpha$-form.

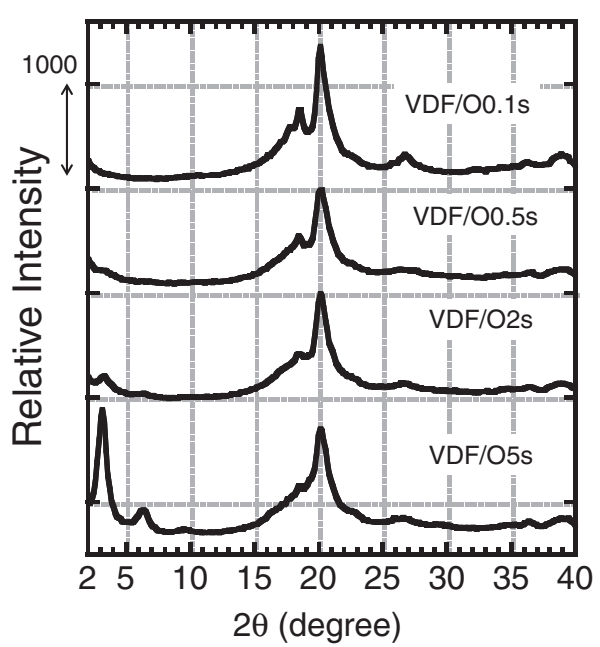

Figure 3. XRD profiles for slowly cooled films in PVDF/Organo-clay nanocomposites with various compositions. Sample notation is described in SAMPLE PREPARATION.

crystal structure. We consider that difference from $2 \theta=20.7^{\circ}$ of the uniaxially stretched PVDF $\beta$-form is modification due to interacting clay content. We do not consider possibility of special case of other crystal form or new crystal form of PVDF for the disagreement between 20.1 and 20.7 degrees. Bulk 


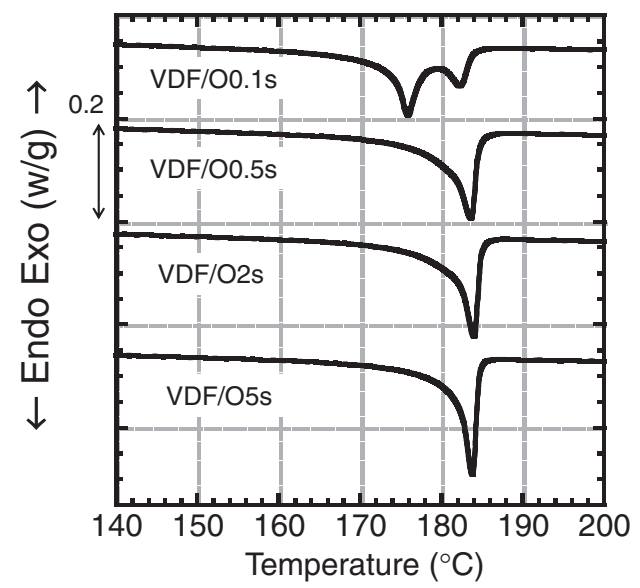

Figure 4. DSC heating curves for slowly cooled films of PVDF/organo-clay nanocomposites with various compositions corresponding to XRD profiles of Figure 3.

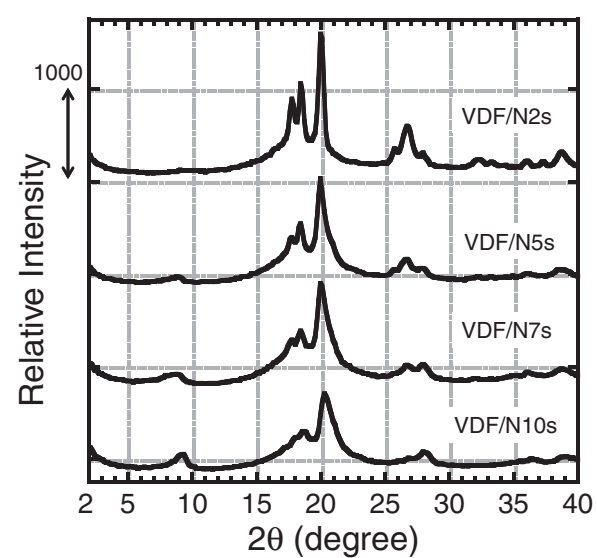

Figure 5. XRD profiles for slowly cooled films of PVDF/natural-clay composites with various compositions. Sample notation is described in SAMPLE PREPARATION.

lattice spacing of the $\beta$-form is easily changed by crystallization treatment such as pressure procedure. ${ }^{16}$ For clay-modified $\beta$-form, we also observed D-E hystersis loop, which is the evidence of oriented all-trans chains causing spontaneous polarization. ${ }^{15}$

Figures 5 and 6 show XRD profiles and DSC heating curves for slowly cooled films of PVDF/natural-clay composites. In $\mathrm{PVDF} /$ natural-clay composites, XRD profile gradually changed to the type of $\beta$-form with increase of natural-clay content. As shown by Figure 6, when we increased natural-clay content, endothermal peak of the $\beta$-form gradually increased and one of the $\alpha$-form decreased.

Natural-clay is hydrophilic. Therefore, natural-clay aggregates to form large clusters due to its insufficient affinity to PVDF matrix. Above results indicate that aggregating naturalclay also cause observable epitaxial growth of the PVDF $\beta$ form crystal when enough content of clay are included. By these results, we confirmed that efficiency of crystallization into the PVDF $\beta$-form depends on both structure and amount of

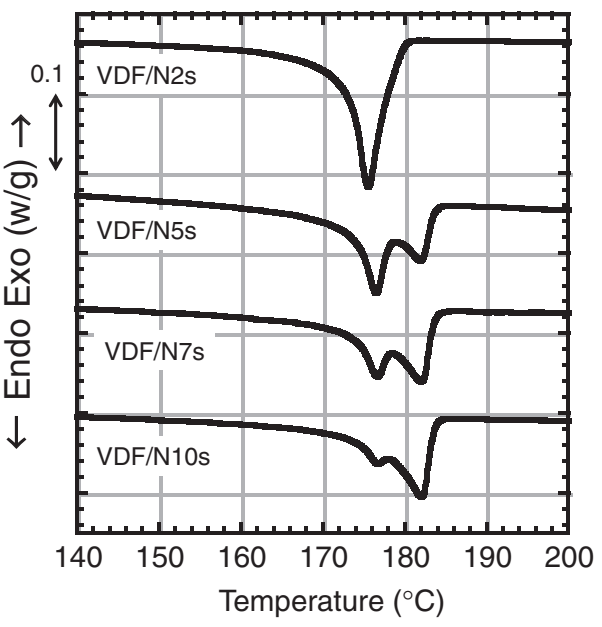

Figure 6. DSC heating curves for slowly cooled films of PVDF/natural-clay composites with various compositions corresponding to XRD profiles of Figure 5 .

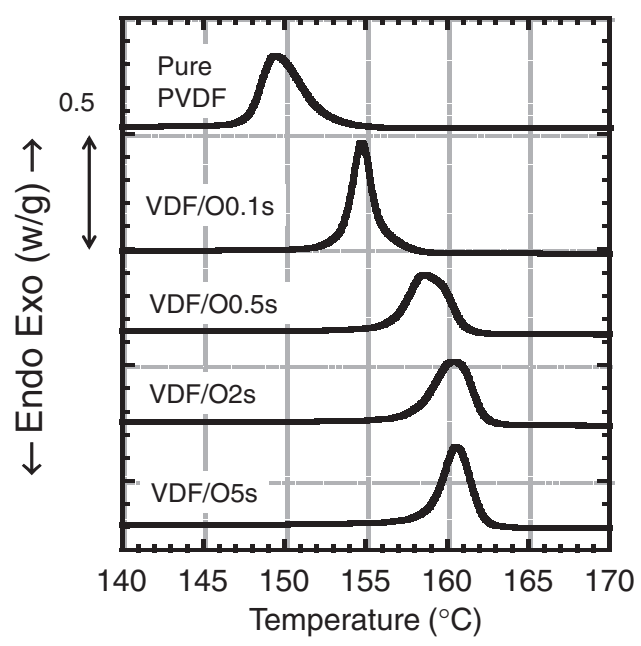

Figure 7. DSC cooling curves at rate $1^{\circ} \mathrm{C} / \mathrm{min}$ of $\mathrm{PVDF} /$ organo-clay nanocomposites with various compositions.

clay surface exposed to the PVDF matrix. XRD profile and DSC heating curve for VDF/O0.1s in Figures 3 and 4 indicates the intermediate data between PVDF $\alpha$-form and VDF/O0.5s. The PVDF crystal structure in VDF/O0.1s indicated both characters of PVDF $\alpha$-form and $\beta$-form. The DSC heating curve indicated the double endothermal peaks at $176.0^{\circ} \mathrm{C}$ and $184.2^{\circ} \mathrm{C}$ which are attributable to the PVDF $\alpha$-form and $\beta$ form, respectively. The results of Figures 3 and 4 of small content of organo-clay were similar to Figures 5 and 6 of natural-clay compositions. This indicates that the difference between organo-clay and natural-clay mainly stems from difference of area of clay surface exposed to PVDF matrix.

Figures 7 and 8 show DSC cooling curves at rate of $1^{\circ} \mathrm{C} /$ min, respectively, for PVDF/organo-clay nanocomposites and $\mathrm{PVDF} /$ natural-clay composites of various weight fractions. We examined this cooling rate of DSC measurement considering the slowly cooled samples. In both cases of PVDF/organo-clay 


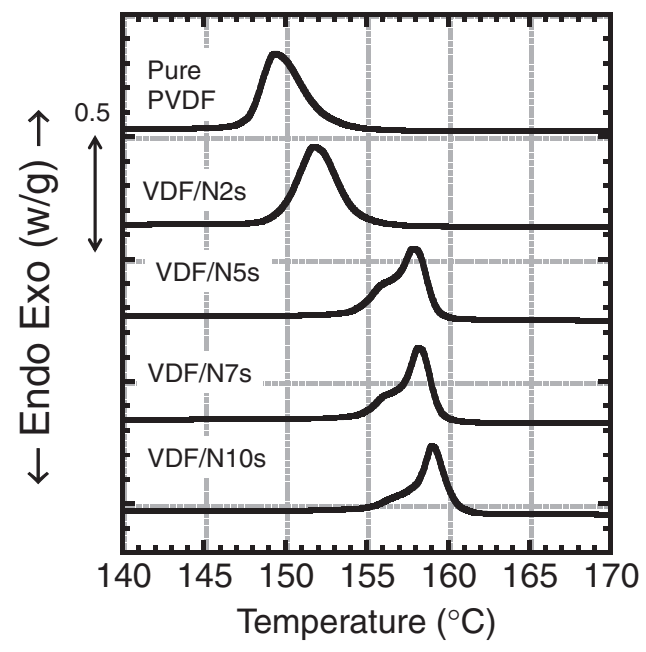

Figure 8. DSC cooling curves at rate $1^{\circ} \mathrm{C} / \mathrm{min}$ of $\mathrm{PVDF} /$ natural-clay composites with various compositions.

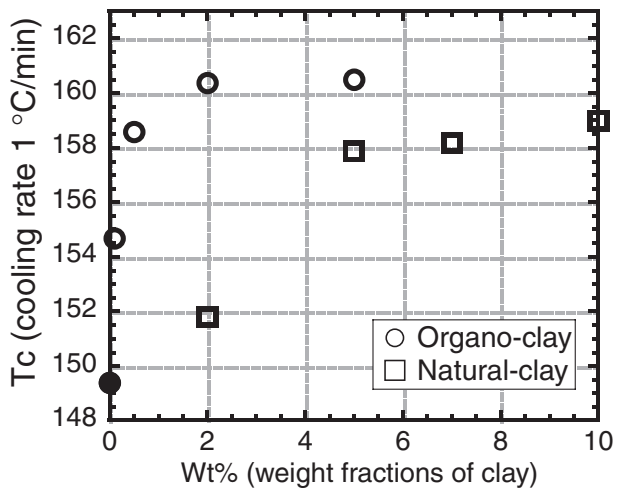

Figure 9. Relation between clay content and crystallization peak temperature $\left(T_{\mathrm{c}}\right)$ at cooling rate of $1^{\circ} \mathrm{C} / \mathrm{min}$. Open circles are of organoclay. Open squares of natural-clay. The closed circle is of pure PVDF.

nanocomposites and PVDF/natural-clay composites, crystallization temperature of PVDF got higher with increase of clay contents. The blended clays acted as nucleating agent for PVDF crystallization.

Figure 9 summaries results of Figures 7 and 8 showing relationship between crystallization peak temperatures of blended organo-clay and natural-clay. As clay content was increased, the crystallization peak temperature increased indicating saturation. Result presented by Figure 9 indicates our conclusion that crystallization behavior depends on amount of clay surface exposed to PVDF matrix.

\section{Quenched Samples}

The present quench treatment in ice water corresponds to much greater degree of supercooling as compared with slowly cooled treatment. Figure 10 shows XRD profiles for quenched films of PVDF/organo-clay nanocomposites. PVDF crystal structure was coexistence of the $\beta$-form of peak at $2 \theta=20.1^{\circ}$ and the $\alpha$-form at $18.6^{\circ}$, as also indicated by slowly cooled

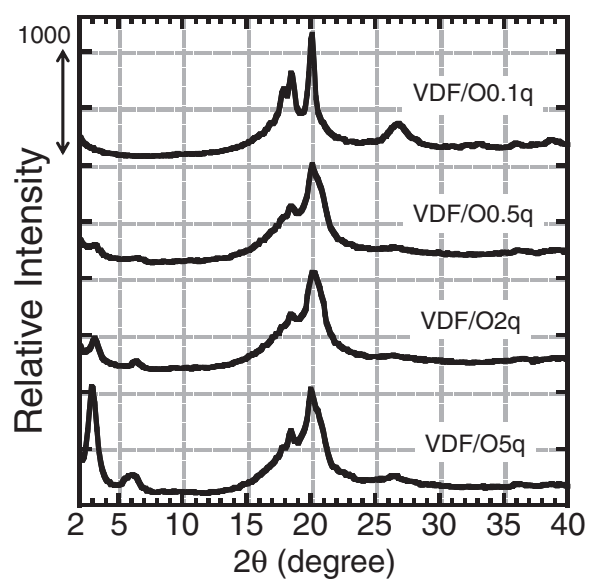

Figure 10. XRD profiles for quenched films in PVDF/organo-clay nanocomposites with various compositions.

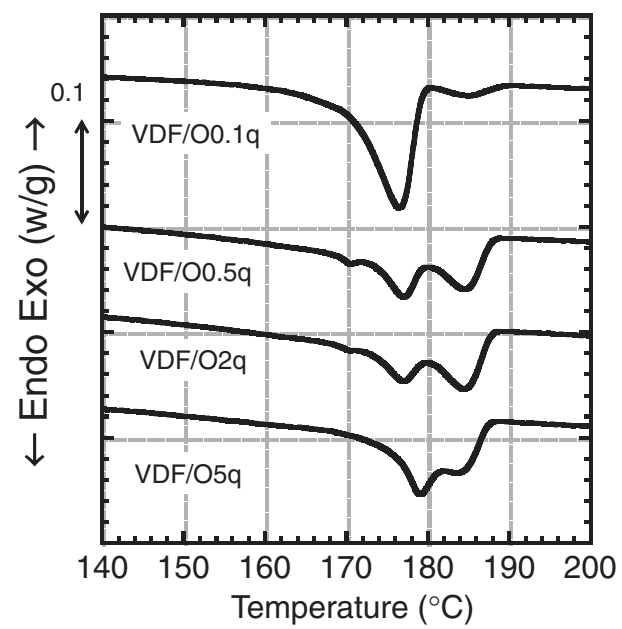

Figure 11. DSC heating curves for quenched films of PVDF/organo-clay nanocomposites with various compositions.

films. Figure 11 shows DSC heating curves at rate of $1{ }^{\circ} \mathrm{C} / \mathrm{min}$ for quenched films of PVDF/Organo-clay nanocomposites. As shown in Figure 11, the double endothermal peaks at around 177 and $184{ }^{\circ} \mathrm{C}$ were indicated. They are attributable to PVDF $\alpha$-form and $\beta$-form, respectively. At Difference of PVDF $\beta$ Form Crystal on the Clay Surface in DISCUSSIONS section, we discuss the difference between a melting temperature of the $\beta$-form of Figure 2 and that of Figure 11 at $184^{\circ} \mathrm{C}$. Comparing results of quenched films and slowly cooled films, it is clear that crystal structure depends on a degree of supercooling. Higher degree of supercooling indicated higher content of $\alpha$ form crystal.

As shown date of VDF/O0.1q in Figures 10 and 11, PVDF crystal structure is in $\alpha$-form from XRD profile, and only single endothermal peak of $\alpha$-form are indicated from DSC heating data.

Figures 12 and 13, respectively, show XRD profiles and DSC heating curves for quenched films of PVDF/natural-clay composites. In Figures 12 and 13, peaks are only of the $\alpha$-form. 


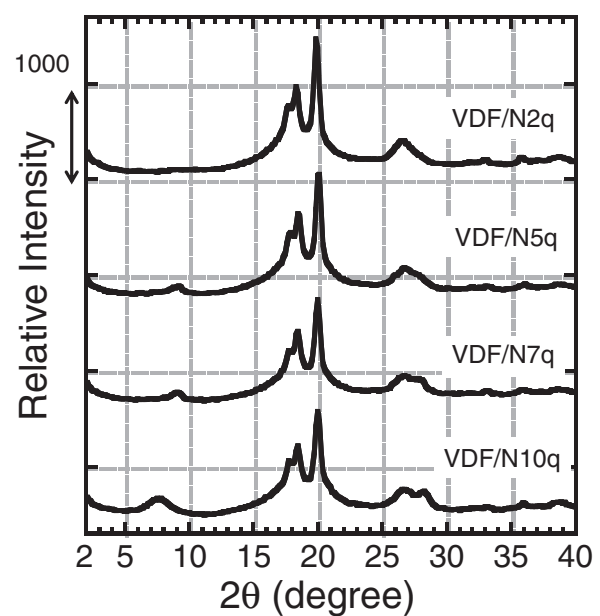

Figure 12. XRD profiles for quenched films of PVDF/natural-clay composites with various compositions.

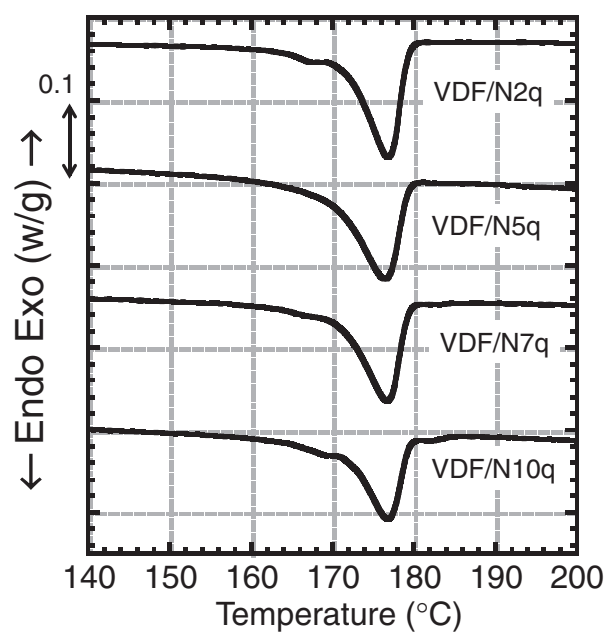

Figure 13. DSC heating curves for quenched films of PVDF/natural-clay composites with various compositions.

\section{DISCUSSIONS}

\section{Epitaxial Growth}

Figure 9 shows that the crystallization peaks of slowly cooled samples depend on amount of clay. It is lower for the natural-clay than for the organo-clay. The natural-clay also works as a nucleating agent when enough amounts are included. Effective area of exposed surface of the natural-clay is smaller than that of the organo-clay because of difference in a degree of dispersion. The shift of crystallization peak to lower temperature for $\mathrm{PVDF} /$ natural-clay composites is explained by smaller amount of exposed surface.

We consider that the role of surface of natural-clay is the same with organo-clay. For the organo-clay, we have suggested in our previous paper that the crystal induction mechanism is epitaxial growth considering in lattice matching and chargedipole interfacial interaction between surface of clay and PVDF all-trans chain in $\beta$-form crystal. ${ }^{15} \mathrm{We}$ conclude that epitaxial growth is induced also on the natural-clay for PVDF $\beta$-form crystal.

\section{Difference of PVDF $\boldsymbol{\beta}$-Form Crystal on the Clay Surface}

Figures 2 and 4 shows that the melting point of $\beta$-form crystal is clearly different between uniaxially stretched PVDF $\beta$-form film and composite films. Observed melting peak was higher for clay-induced $\beta$-crystal than for stretch-induced $\beta$ form crystal.

Similar change in melting point of the $\beta$-form crystal was also reported for high pressure treatment of PVDF. ${ }^{16-19}$ Matsushige and Takemura considered that the $\beta$-form crystal domains in mechanically stretched film are very tiny size compared to samples treated in high pressure. Ohigashi and Hattori mentioned that the melting point of PVDF $\beta$-form crystal increases up to $207^{\circ} \mathrm{C}$ when thick lamella grows. ${ }^{16,18,19}$ Their conclusions are that lower melting temperature of stretched $\beta$-form crystal originates from defects mainly consisting of trans-gauche (TG) sequences in extended all-trans chains. ${ }^{16,17}$

We consider that generation of defects that contains TG sequences is suppressed for clay-induced PVDF $\beta$-crystal on surface of the clay platelet. This is the main reason of the higher melting temperature.

PVDF Crystallization in Clay Composite for Slow Cooling

Slowly cooled samples showed a case in which single melting peak appeared for heating DSC, while X-ray pattern indicated coexisting $\alpha$ - and $\beta$-crystals. Results of Figure 3 of organo-clay shows decreasing $\alpha$-peak due to increase of clay content, while corresponding Figure 4 shows single melting peaks with a shoulder except for the case of VDF/O0.1s. Difference is remarkable when we compare it with Figures 5 and 6 of natural-clay and Figure 11 of quenching of organoclay composite. Double melting peaks of slowly cooled samples of organo-clay composite appeared for Figure 4 for small content of the clay and Figure 6 for natural-clay.

Wang and co-workers showed that melt-spun PVDF fibers at spinning stresses at $3 \mathrm{MPa}$ are polymorphic including both $\alpha$ and $\beta$-form crystals. $^{20,21}$ The crystal structure in their case is considered as shish-kebabs structure with shish of extended chains of $\beta$-crystal and kebabs of lamellar crystal of $\alpha$ crystal. ${ }^{20,21}$ In crystallization process, $\beta$-crystal first crystallizes into a type of extended chains in a spinning field, and then after that the lamellar $\alpha$-crystal is attached onto the extended chains. In addition, the melting point of the $\beta$-crystal in PVDF fibers is higher than that of $\alpha$-form crystal. ${ }^{20}$

We expect that behavior of PVDF crystallization on the clay surface is similar to the above case in spinning field of PVDF fiber processing. An extensional force is expected to PVDF chain on the surface of clay platelet during epitaxial growth by lattice matching and charged-dipole interfacial interaction. The $\beta$-form crystal first crystallizes on the surface. After that lamellar crystals of $\alpha$-form are attached onto the $\beta$-form crystal. The melting point of $\alpha$-crystal is affected near the surface induced $\beta$-crystal causing higher value compared to the 
bulk $\alpha$-crystal. The melting peak merges to that of the surface induced $\beta$-crystal in the case occupied by clay component. This is the reason for the single melting peak of VDF/O0.5s, VDF/ $\mathrm{O} 2 \mathrm{~s}$, and VDF/O5s of Figure 4.

Maiti and Okamoto reported for Nylon-6 crystal in Nylon-6/clay nanocomposites. ${ }^{22}$ They indicated that crystal of Nylon-6 grows epitaxially on the surface of clay platelet, and the structure is the typical "shish-kebabs type" by observing electron transmission micrograph images.

\section{PVDF Crystallization in Quenched Composite}

For quenched samples we see two types of DSC curves. One is for Figure 11 of the PVDF/organo-clay composites with $0.5,2$, and $5 \mathrm{wt} \%$ showing the double melting peaks that correspond to $\alpha$ - and $\beta$-form crystals. The other is $0.1 \mathrm{wt} \%$ of Figure 11 for small content of the organo-clay and natural-clay composites of Figure 13 showing the single melting peak of $\alpha$-form crystal.

In the case of quenched treatment, the $\alpha$-form crystal is easily nucleated in amorphous bulk region at much lower temperature compared to the equilibrium melting temperature. The $\beta$-form crystal is also formed on the clay surface. However, if effective content of clay surface is small, growth of surface-generated $\beta$-crystal is dominated by bulk-generated $\alpha$-crystal. Nucleation is easily occurs at low crystallization temperature, because the critical nucleus size and the free energy barrier are decreasing quantities of degree of supercooling. In the case of quenched treatment, nucleation of the $\alpha$ crystal could occur at everywhere in bulk amorphous region. When PVDF nucleation and growth occur at the amorphous region, crystal is the $\alpha$-form crystal. Potential energy of the $\alpha$-form crystal is the lowest in PVDF polymorphisms at atmospheric pressure. $^{2}$

\section{CONCLUSION}

PVDF also crystallize into $\beta$-form in PVDF/natural-clay composites. This result is one of the important evidence for that the crystallization mechanism is the epitaxial growth of the PVDF $\beta$-form crystal on the surface of clay platelets. In this our experience, PVDF crystallization behavior depends on amount of clay surface exposed to PVDF matrix and a degree of supercooling. Induced structures did not show remarkable qualitative difference between organo-clay and natural-clay.

DISCUSSIONS also show following conclusions. The difference of melting points between stretch-induced $\beta$-crystal and clay-induced $\beta$-crystal indicates that formation of defects such as TG sequences is suppressed for PVDF $\beta$-form crystal on the surface of clay platelet. We expect that PVDF crystallization on the surface of clay platelet is almost the same phenomena with that in PVDF fiber processing. Role of epitaxial field is similar to extension field. An extension stress is induced by charge-dipole interfacial interaction in lattice matching process.

Acknowledgment. The authors would like to thank Mr. N. Yanagisawa, Mr. H. Komatsuzaki, Mr. S. Sakairi and Mr. T. Kimu for assistance in the experiments, and Dr. D. Fukuzawa, Mr. H. Shinohara who give our this study good points with the results of their study. This work was supported by Grants-inAid from the Ministry of Education, Culture, Sports, Science and Technology of Japan, No. 18068003 (Priority Area "Soft Matter Physics") and No. 17310058.

Received: November 18, 2008 Accepted: January 22, 2009 Published: March 18, 2009

\section{REFERENCES}

1. R. Hasegawa, M. Kobayashi, Y. Chatani, and H. Tadokoro, Polym. J., 3, 600 (1972).

2. R. Hasegawa, M. Kobayashi, and H. Tadokoro, Polym. J., 3, 591 (1972).

3. Y. Takahashi, Y. Matsubara, and H. Tadokoro, Macromolecules, 15, 334 (1982)

4. M. Kobayashi, K. Tashiro, and H. Tadokoro, Macromolecules, 8, 158 (1975).

5. H. S. Nalwa, "Ferroelectric Polymers: Chemistry, Physics and Applications," Marcel Dekker, New York, 1995, p. 67.

6. T. Takahashi, M. Date, and E. Fukada, Ferroelectrics, 32, 73 (1981).

7. T. Furukawa, M. Date, and E. Fukada, J. Appl. Phys., 51, 1135 (1980).

8. M. Tamura, K. Ogasawara, N. Ono, and S. Hagiwara, J. Appl. Phys., 45, 3768 (1974).

9. T. Furukawa and G. E. Johnson, Appl. Phys. Lett., 38, 1027 (1981).

10. R. G. Kepler and R. A. Anderson, J. Appl. Phys., 49, 1232 (1978).

11. D. Naegele and D. Y. Yoon, Appl. Phys. Lett., 33, 132 (1978).

12. L. Priya and J. P. Jog, J. Polym. Sci., Part B: Polym. Phys., 40, 1682 (2002).

13. L. Priya and J. P. Jog, J. Polym. Sci., Part B: Polym. Phys., 41, 31 (2003).

14. L. Priya and J. P. Jog, J. Appl. Polym. Sci., 89, 2036 (2003).

15. E. Yamada, A. Nishioka, H. Suzuki, T. Koda, and S. Ikeda, J. Appl. Phys., 46, 7371 (2007).

16. H. Ohigashi and T. Hattori, Jpn. J. Appl. Phys., 28, L1612 (1989).

17. K. Matsushige and T. Takemura, J. Polym. Sci., Polym. Phys. Ed., 18, 1665 (1980).

18. T. Hattori, M. Kanaoka, and H. Ohigashi, J. Appl. Phys., 79, 2016 (1996).

19. T. Hattori, M. Hikosaka, and H. Ohigashi, Polymer, 37, 85 (1996).

20. Y. Wang, M. Cakmak, and J. L. White, J. Appl. Polym. Sci., 30, 2615 (1985).

21. M. Cakmak, A. Teitge, H. G. Zachmann, and J. L. White, J. Polym. Sci., Part B: Polym. Phys., 31, 371 (1993).

22. P. Maiti and M. Okamoto, Macromol. Mater. Eng., 288, 440 (2003). 